\title{
Tierexperimentelle Untersuchungen zum Problem der Insulinresistenz bei Adipositas und Diabetes mellitus. Pathogenese des fettsüchtig-hyperglykämischen Syndroms1, 2)
}

\author{
Von H. Huchzermeyer, K. H. Rudorff und W. Starb \\ Diabetes-Forscbungsinstitut und Institut für Pbysiologische Chemie II der Universität Diisseldorf
}

(Eingegangen am 15. Dezember 1972/16. März 1973)

Herrn Prof. Dr. Dr. Ernst Schïtte zum 65. Geburtstag gewidmet

Bei der New Zealand obese mouse (NZO) verschlechtert sich die Glucosetoleranz kontinuierlich mit zunehmendem Alter und Körpergewicht bei gleichzeitigem Ȧnstieg der Serumlipide. Gewichtsreduktion verbessert Glucosetoleranz und Serumlipide. In vivo besteht eine deutliche Insulinresistenz, Insulindosen bis zu $20 \mathrm{IE} / \mathrm{kg}$ Körpergewicht i. p. lösen keine Krämpfe aus. Der Blutzucker zeigt nach i. p. Injektion von 0,5 IE/kg Körpergeuricht bei Gewichtszunahme nicht mehr einen Abfall, sondern vielmehr einen paradoxen Anstieg. In der isoliert perfundierten Leber der NZO-Maus bestcht, verglichen mit der swiss albino Maus, keine gesteigerte Gluconeogenese aus L-Alanin. Insulin hemmt im Gegensatz zu Ratte und swiss albino Maus an der isoliert perfundierten Leber der NZO-Maus vor und nach Gewichtsreduktion nicht die Gluconeogenese aus L-Alanin. Ebenso fehlt ein protein-anaboler Effekt des Insulins.

Analog zur Leber wiesen Diaphragma, Myocard und epididymales Fettgewebe mit steigendem Alter eine weitgehende Insulinresistenz in vitro auf. Allerdings zeichnet sich das Fettgewebe durch mehrere Besonderheiten aus. Einmal ist nach Gewichtsreduktion nur am Fettgewebe eine erhöhte Insulinsensitivität nachzuweisen, zum anderen findet sich am Fettgewebe eine regelmäßige Stimulation der Lipidsynthese aus Glucose durch Insulin. Der letztere Befund scheint ein wesentlicher Faktor in der Pathogenese der metabolischen Fettsucht der NZO-Maus zu sein. Eine verzögerte Oxidationsrate von Stearinsäure konnte dagegen nicht nachgewiesen werden.

Für die Entwicklung des hyperglykämischen Syndroms der NZO-Maus sind neben humoral-metabolischen Faktoren die beschriebene weitgehende Insulinresistenz von Fettgewebe, Muskulatur und Leber verantwortlich. Eine gesteigerte Gluconeogenese - gemessen an Leberenzymaktivitäten in vivo, an der Gluconeogenese aus $\mathrm{X}$-Alanin in der isoliert perfundierten Leber und ar der Glucoseproduktion von Leberschnittpräparationen - spielt dagegen keine oder nur eine geringe Rolle. Úberträgt man dieses Tiermodell der NZO-Maus auf den Menschen, so weist es Analogien zum adipösen Patienten mit gestörter Glucosetoleranz und Hyperinsulinismus, aber noch ohne Erschörpfung der Inselzellfunktion, auf.

\section{Experimental studies on the problem of insulin resistance in Adipositas and Diabetes mellitus, with the aid of New Zealand obese mice. Patbogenesis of the obese-byperglycaemic syndrome}

In New Zealand obese mice (NZO) glucose tolerance deteriorates with increasing age and weight, with the simultaneous elevation of serum lipids. Loss of weight is found to improve glucose tolerance and the concentration of the serum lipids. There is a marked insulin resistance in vivo; insulin doses up to $20 \mathrm{IU} / \mathrm{kg}$ body weight, administered $\mathrm{i}$. p., do not produce any spasm. With increasing weight, however, after i. p. injection of $0.5 \mathrm{IU} / \mathrm{kg}$ body weight, the blood sugar no longer showrs a decrease but a paradoxical elevation.

In the isolated perfused liver of the NZO-mouse, no increase of $\mathrm{L}$-alanine gluconeogenesis occurs as compared with Swiss albino mice. Contrary to rats and Swiss albino mice, insulio does not suppress the $\mathrm{L}$-alanine gluconeogenesis in the isolated perfused liver of the $\mathrm{NZO}$ mouse before and after weight reduction. Furthermore, a protein-anabolic effect of insulin is also absent.

Like the liver, the diaphragm, myocardium and epididymal adipose tissue also show an increased insulin resistance consistent with advancing age. However, the adipose tissue reacts differently: On the one hand, an increased insulin sensitivity is demonstrable following weight reduction, on the other, a regular stimulation of lipid synthesis from glucose is effected by insulin. The latter result appears to be an important factor in the pathogenesis of metabolic obesity in the NZO mouse although a delayed oxidation rate of stearic acid could not be demonstrated.

Apart from humoral-metabolic factors, the described general insulin resistance of adipose tissue, musculature and liver is responsible for development of the hyperglycaemic syndrome in NZO mice. An increased gluconeogenesis, measured according to enzymatic activity of the liver in vivo, L-alanine gluconeogenesis of isolated perfused liver and glucose production of liver slices, play only a slight role, if any. Relating this study on NZO mice to humans, an analogy to obese patients with impaired glucose tolerance and hyperinsulinism is apparent, although the islet cells are still active in the latter.

Die Fettsucht ist eine der wesentlichsten Faktoren für die Manifestation eines Diabetes mellitus. Etwa $80 \%$ der frisch entdeckten Altersdiabetiker sind oder waren übergewichtig $(1,2)$ und bei über $50 \%$ der Fettsüchtigen ist die Glucosetoleranz gestört $(1,3)$. Ursache dieser Störung der Glucoseutilisation ist aber nicht ein absoluter Insulinmangel. Vielmehr sind bei Adipösen das immunologisch meßbare Seruminsulin und die insulinähnliche Aktivität erhöht $(1,3)$. Da dieser
Hyperinsulinismus in der Regel aber keine Hypoglykämie bedingt, muß eine Insulinunterempfindlichkeit vorliegen. Als mögliche Ursachen dieser Insulinresistenz werden

1. humorale Faktoren (antagonistische Hormone, Stoffwechselprodukte, Antikörper usw.),

1) Auszugsweise vorgetragen auf dem 6. Kongreß der Deutschen Diabetes-Gesellschaft Düsseldorf, 14. und 15. Mai 1971.

2) Mit Unterstützung der Deutschen Forschungsgemcinschaft. 
2. eine gestörte Permeabilität der Kapillarwand, möglicherweise aufgrund der Verdickung der Basalmembran und

3. zelluläre Faktoren diskutiert (4).

Das Auftreten von Fettsucht, Hyperglykämie und Hyperinsulinismus konnte in den letzten Jahren ebenso an über 10 Kleintierstämmen beobachtet werden. Zahlreiche Analogien zum menschlichen Reifediabetes konnten aus der Bearbeitung von Teilfragen an diesen Tieren gewonnen werden. In früheren Arbeiten haben wir die New Zealand obese Maus (NZO) (5) als einen Vertreter dieser Tierstämme zu charakterisieren versucht $(6-8,10)$. Dieser Inzuchtstamm zeichnet sich durch ein rezessiv hereditäres, permanentes, benignes, fettsüchtig-hyperglykämisches Syndrom ohne Neigung zur Ketoacidose bei gleichzeitiger relativer Insulinresistenz und Hyperinsulinämie aus. Wir führten nun zur Pathogenese der gestörten Glucosetoleranz, zur Insulinsensitivität in vivo und von Muskulatur, Fettgewebe und Leber in vitro und zur Beeinflussung der Befunde durch Gewichtsreduktion weitere Untersuchungen durch und haben im Anschluß daran versucht, die gewonnenen tierexperimentellen Ergebnisse auf den Menschen zu übertragen.

\section{Material und Methoden}

$\mathrm{Zu}$ den Versuchen wurden männliche NZO-Mäuse (5) verwendet und die Ergebnisse mit früheren Befunden an swiss albino Mäusen und Wistar-II-Ratten verglichen $(6,8,9,10)$. Bei konstanten Umweltbedingungen hatten die Tiere freien Zugang $\mathrm{zu}$ Wasser und Futter (Standardkost, Höveler, Langenfeld-Immigrath). Zur Erzielung einer Gewichtsreduktion wurde den 8-9 Monate alten NZO-Mäusen für 6 Wochen $2,5 \mathrm{~g}$ Standardkost pro Tag gegeben. Vor den Perfusions- bzw. Inkubationsversuchen hungerten die Tiere $24 \mathrm{~h}$.

Zur Durchführung der Glucosetoleranz-Tests wurden den gefütterten Tieren jeweils um 8 Uhr $2,5 \mathrm{~g}$ Glucose (vgl. $6,11,12$ ) pro $\mathrm{kg}$ Körpergewicht in $100 \mathrm{~g} / \mathrm{l}$ Lösung intraperitoneal injiziert und mit Versuchsbeginn das Futter entzogen. Nach 20,60, 120 und $180 \mathrm{~min}$ wurde aus der Schwanzspitze Blut zur Glucosebestimmung entnommen, der Harn wurde nicht auf eine Glucoseausscheidung geprüft.

Die Methodik der Leberperfusion ist in früheren Arbeiten ausführlich beschrieben $(9,10)$. Die Entnahme der Leber erfolgte in Evipan-Narkose $(20 \mathrm{mg} / 100 \mathrm{~g}$ Körpergewicht i. p.) unter Zusatz von $0,1 \mathrm{ml}$ Liquemin (500 U. S. P.-E. Heparin). Nach einem anoxischen Intervall von weniger als $5 \mathrm{~min}$ wurde die Leber $90 \mathrm{~min}$ lang kontinuierlich und zyklisch in einer modifizierten MILLER'schen Apparatur mit $50 \mathrm{ml}$ Krebs-Ringer-Bicarbonatlösung ( $\mathrm{pH} 7,4)$ mit $2 \mathrm{~g}$ Albumin, $10 \mathrm{~g}$ Hämoglobin in Form gewaschener Rindererythrocyten und 5000 U. S. P.-E. Heparin auf $100 \mathrm{ml}$ Medium perfundiert. Nach $25 \mathrm{~min}$ Vorperfusion wurden $10 \mathrm{mmol} / 1 \mathrm{~L}$-Alanin, $5 \mathrm{~min}$ später $1 \mu \mathrm{Ci}$ [U-14 $\mathrm{C}$ L-Alanin

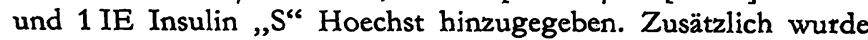
1 IE Insulin "S" Hoechst pro Stunde infundiert. Im Perfusat wurden nach 30 und 90 min Glucose, Harnstoff und Ketonkörper bestimmt, nach $90 \mathrm{~min}$ außerdem der ${ }^{14} \mathrm{C}$-Einbau aus [U-14 $\mathrm{C}] \mathrm{L}-\mathrm{Alanin}$ in Plasmaprotein und in Protein und Gesamtlipide der Leber. ${ }^{14} \mathrm{CO}_{2}$ wurde in den Zeiträumen 0-30 min und 30-60 min nach Zugabe von [U-14 C]L-Alanin aufgefangen.

Die Insulinsensitivität in vivo prüften wir mit intraperitonealen Gaben von $0,5-20$ IE Insulin Hoechst pro $\mathrm{kg}$ Körpergewicht. In vitro wurden in Anwesenheit oder Abwesenheit von Insulin $(1 \mathrm{mU} / \mathrm{ml}$ Insulin Hoechst) jeweils $100-150 \mathrm{mg}$ schwere Gewebspräparationen (Schnitte) von epididymalem Fettgewebe,
Diaphragma und Herzmuskel in $2 \mathrm{ml}$ Krebs-Ringer-Hydrogencarbonatlösung ( $\mathrm{pH} 7,4)$ unter Zusatz von Albumin $(2 \mathrm{mg} / \mathrm{ml}$ ), [U-11 C]Glucose $(0,5 \mu \mathrm{Ci} / \mathrm{ml})$ und Glucose $(2 \mathrm{mg} / \mathrm{ml}) 2 \mathrm{~h}$ bei $37^{\circ} \mathrm{C} \mathrm{im}$. Schüttelbad inkubiert. Die Reaktion wurde gestoppt mit $0,2 \mathrm{ml} \mathrm{H}_{2} \mathrm{SO}_{4}(100 \mathrm{~g} / \mathrm{kg})$. Gemessen wurden die Glucoseaufnahme aus dem Medium, die ${ }^{14} \mathrm{CO}_{2}$-Bildung und der ${ }^{14} \mathrm{C}$-Einbau aus [U-14 C] Glucose in die Gesamtlipide und das Glykogen.

Die Glucosebestimmungen der Glucosetoleranz-Tests erfolgten nach der o-Toluidin-Methode im Autoanalyzer (13). Im Perfusat wurden Glucose nach der Glucoseoxidase-Methode, Harnstoff enzymatisch mit Urease (Biochemica-Test-Combinationen, Boehringer, Mannheim), Acetessigsäure und $D(-) \beta$-Hydroxybuttersäure nach STEIN und BÄSsLER (14) analysiert. [U_14C] Glucose wurde als Phenylosazon isoliert und nach dex Methode von RuDORFF et al. (9) bestimmt. ${ }^{14} \mathrm{CO}_{2}$ wurde in einem Gemisch aus Äthanolamin und Äthylenglykolmonomethyläther im Verhältnis 1:2 absorbiert und gemessen. Aufarbeitung und Aktivitätsmessung der Plasma- und Leberproteine erfolgte nach MCLEAN et al. (15), der Gesamtlipide nach Folch et al. (16), des Glykogens nach WaLAAS und WaLAAS (17).

Zur Bestimmung der Serumlipide wurden folgende Methoden benutzt: Gesamtlipide, Gesamt-Cholesterin und verestertes Cholesterin mit den Biochemica-Tèst-Combinationen (Boehringer, Mannheim), Gesamt-Esterfettsäuren nach RosenTHAI et al. (18), Glycerin und Triglyceride nach KREUTz (19) und freie Fettsäuren nach ITAYA und UI (20).

Die Aktivitätsmessungen erfolgten im Tri-Carb Liquid Scintillation Spectrometer der Firma Packard Instruments. [U-14 C]LAlanin, $\left[\mathrm{U}-{ }^{14} \mathrm{C}\right]$ Stearinsäure und $\left[\mathrm{U}-{ }^{14} \mathrm{C}\right] \mathrm{Glucose}$ wurden bei The Radiochemical Centre, Amersham (England), die verwendeten Enzyme und übrigen Substrate bei den Firmen Merck, Darmstadt und Boehringer, Mannheim gekauft. Als Parameter der einzelnen Probekollektive sind im Text, in den Tabellen und Abbildungen neben dem arithmetischen Mittelwert die Standardabweichungen der Mittelwerte $(\overline{\mathbf{x}} \pm s \overline{\mathrm{x}})$ angegeben. Die Signifikanzpprüfungen erfolgten mit dem t-Test nach STUdent.

\section{Ergebnisse und Diskussion}

\section{Glucosetoleranz und Insulinresistenz}

Im Vergleich zur stoffwechselgesunden swiss-albinoMaus ist die Glucosetoleranz der NZO-Maus gestört und nimmt mit zunehmendem Alter und Gewicht progressiv ab $(6,11)$. Bereits bei Gewichtskonstanz bessert sich diese Glucoseverwertung (6), aber die Besserung ist noch deutlicher nach Gewichtsabnahme mit Hilfe einer 6-wöchigen Reduktionskost. So konnte das Gewicht der 8-9 Monate alten Tiere von $58 \mathrm{~g}$ auf $39 \mathrm{~g}$ reduziert und die Toleranz dadurch wesentlich gebessert werden (Abb. 1). Dies entspricht Beobachtungen bei Mensch und Tier, daß sich Glucosetoleranz aber auch Serumlipide, Insulinspiegel und Insulinsekretion nach Reduktion des Ubergewichtes normalisieren (vgl. 1). Weiterhin zeigen die NZO-Mäuse analog zu Befunden bei menschlicher Adipositas (21) eine relative Insulinresistenz. Während 6 Wochen alte Tiere nach intraperitonealer Injektion von $0,5 \mathrm{IE}$ Insulin Hoechst/kg Körpergewicht normal mit einem Blutzuckerabfall reagierten, stieg der Blutzucker bei den 8 Monate alten Mäusen sogar an, eine Beobachtung, die auch BIELsChowsKY und BIELSCHOWSKY (22) machten. Und auch noch nach Gewichtsreduktion zeigt sich diese, wenn auch etwas geringer, paradoxe Insulinwirkung (Abb. 2). Eine ausreichende Erklärung für dieses Phänomen können wir zur Zeit nicht geben. Eine reaktive Hyperglykämie nach kurzfristigem Blut- 


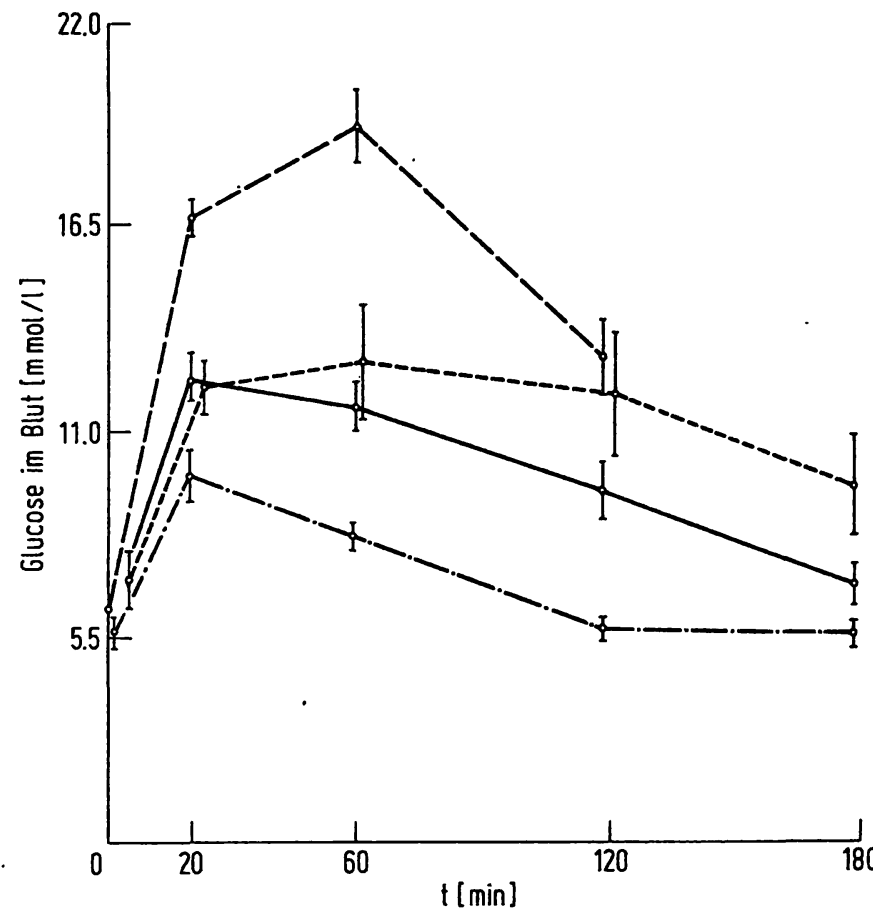

Abb. 1

Glucosetoleranz-Test $(2,5 \mathrm{~g} / \mathrm{kg}$ Körpergewicht i. p.) bei männlichen NZO-Mäusen von 2 Monaten $(--$ Gewicht $34,2 \pm 2,15 \mathrm{~g}, \mathrm{n}=10)$, 8 -9 Monaten (- - Gewicht $58,06 \pm 1,46 \mathrm{~g}, \mathrm{n}=16), 9-10$ Monaten $8-9$ Monaten $(--G)-G e w i c h t 58,06 \pm 1,46 \mathrm{~g}, \mathrm{n}=16), 9-10$ Monaten
$(-$ Gewicht $39,06 \pm 1,03 \mathrm{~g}$, nach 6 Wochen Reduktionskost von $2,5 \mathrm{~g}$ Standardkost/Tag $\mathrm{n}=16$ im Vergleich zur weißen Maus $(-\cdot-\cdot-$ Gewicht $42,4 \pm 1,5 \mathrm{~g}, \mathrm{n}=10)$

zuckerabfall in den ersten 10 min konnte jedoch ausgeschlossen werden.

Diese Resistenz gegenüber Insulin - wir konnten insgesamt bis zu $20 \mathrm{IE}$ Hoechst Insulin/kg Körpergewicht i. p. injizieren, ohne Krämpfe auszulösen stellt die Frage nach der Natur des oder der Insulinantagonisten. So vermuteten BIELSCHOwSKY und BIELschowsKY (22) eine Hypersekretion von STH bei der NZO-Maus, eine Annahme, die sich durch radioimmunologische Bestimmung des STH im Plasma nicht bestätigen ließ (23). Ebenso lassen sich im Sinne des „glucose-fatty acid-cycle“ (24) erhöhte Serumspiegel von freien Fettsäuren, aber auch von Triglyceriden und Ketonkörpern als Ursache der Insulinresistenz

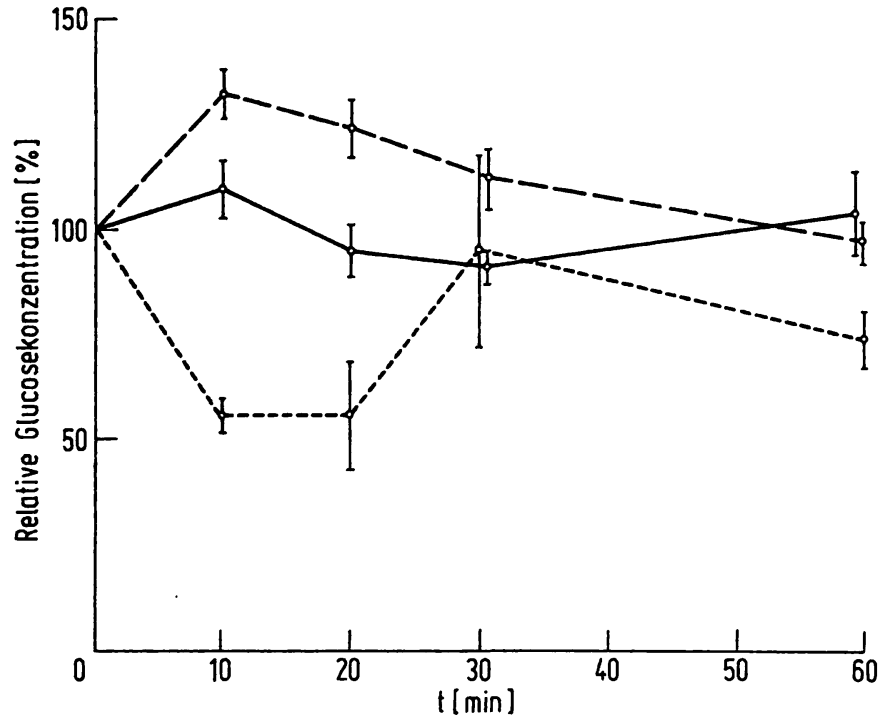

Abb. 2

Einfluß von Insulin $(0,5 \mathrm{E} / \mathrm{kg}$ Körpergewicht i. p.) auf das Verhalten des Blutzuckers männlicher NZO-Mäuse im Alter von 6 Wochen (-- Gewicht 23,2 $\pm 1,15 \mathrm{~g}, \mathrm{n}=6$ ), 8 Monaten (- - Gewicht 46,3 $14 \mathrm{~g}, \mathrm{n}=9$ ) und 9 Monaten ( Gewicht $36,5 \pm 1,4 \mathrm{~g}$ nach 6 Wochen Reduktionskost von $2,5 \mathrm{~g}$ Standardkost/Tag, $\mathrm{n}=8$. Veränderungen sind in $\%$ des Ausgangswertes $=100 \%$ angegeben die Ausgangswerte betrugen: 6 Wochen $=5.9 \pm 0.4 \mathrm{mmol} / \mathrm{l} ; 8 \mathrm{Mo}$ nate $=7,1 \pm 0,7 \mathrm{mmol} / 1 ; 9$ Monate $=6,9 \pm 0,5 \mathrm{mmol} / 1$

diskutieren, die die Glucoseutilisation und Insulinwirkung in der Peripherie verringern sollen. Die Überprüfung der Blutfette von NZO-Mäusen im Alter von $11 / 2-2$ Monaten und 9 Monaten zeigt einen solchen signifikanten Anstieg von Gesamtlipiden, Cholesterin, Triglyceriden, freiem Glycerin und freien Fettsäuren (Tab. 1). Die Ketonkörperproduktion ist dagegen in den ersten 9 Lebensmonaten konstant niedrig (6). Nach Gewichtsreduktion der 9 Monate alten Tiere hatten sich die Lipide, gemessen an Triglyceriden, freiem Glycerin und Gesamtcholesterin, normalisiert.

Einflu $B$ von Insulin auf den Alaninstoffwechsel der isoliert perfundierten Leber

Um bei der Untersuchung einer zell- und gewebsgebundenen Insulinresistenz einen möglichen Effekt jeglicher humoraler Faktoren auszuschalten, unter-

Tab. 1

Serumlipide von NZO-Mäusen (gefüttert) im Alter von $1 \frac{1 / 2}{2} 2$ und 9 Monaten $(\bar{x} \pm s \bar{x})$

\begin{tabular}{|c|c|c|c|c|c|}
\hline & . & & & & \\
\hline & & & $11 / 2-2$ Monate & 9 Monate & \\
\hline & Gesamt-Lipide & $\mathrm{g} / 1$ & $\begin{array}{c}8,95 \pm 0,17 \\
n=6\end{array}$ & $\begin{aligned} 12,32 & \pm 0,19 \\
n & =6\end{aligned}$ & $p<0,0005$ \\
\hline & Gesamt-Cholesterin & $\mathrm{mmol} / 1$ & $\begin{array}{c}4,16 \pm 0,07 \\
n=6\end{array}$ & $\begin{array}{c}12,51 \pm 0,42 \\
n=6\end{array}$ & $\mathrm{p}<0,0005$ \\
\hline & Freies Cholesterin & $\mathrm{mmol} / 1$ & $\begin{array}{c}0,93 \pm 0,18 \\
n=7\end{array}$ & $\begin{array}{c}2,93 \pm 0,17 \\
n=6\end{array}$ & $p<0,0005$ \\
\hline & Gesamt-Esterfettsäuren & $\mathrm{mmol} / \mathrm{l}$ & $\begin{array}{c}12,1 \pm 0,30 \\
n=7\end{array}$ & $\begin{array}{c}12,30 \pm 0,50 \\
n=6\end{array}$ & $\mathbf{p}=\mathbf{n} . \mathbf{s}$ \\
\hline & Glycerid-glycerol (Triglyceride) & $\mathrm{mmol} / \mathrm{l}$ & $9,28 \pm 1,80$ & $14,90 \pm 2,05$ & $p<0,05$ \\
\hline • & Freie Fettsãuren & $\mathrm{mmol} / \mathrm{l}$ & $\begin{array}{c}n=7 \\
0,48 \pm 0,03 \\
n=6\end{array}$ & $\begin{aligned} n & =6 \\
0,62 & \pm 0,03 \\
n & =7\end{aligned}$ & $p<0,005$ \\
\hline & Freies Glycerin & $\mathrm{mmol} / \mathrm{I}$ & $\begin{array}{c}0,26 \pm 0,02 \\
n=5\end{array}$ & $\begin{aligned} 0,39 & \pm 0,04 \\
n & =6\end{aligned}$ & $p<0,01$ \\
\hline
\end{tabular}


Abb. 3 Einfluß von Insulin auf die Glucoseproduktion aus
$.10 \mathrm{mmol} / \mathrm{l}$ L-Alanin in der isoliert perfundierten Leber von normaler und alloxandiabetischer Ratte, swiss albino Maus, NZO-Maus ( $3^{1}$ - 4 Monate) und NZO-Maus $(9-10$ Monate, Gewichtsreduktion). n. s. = nicht signifikant

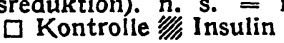

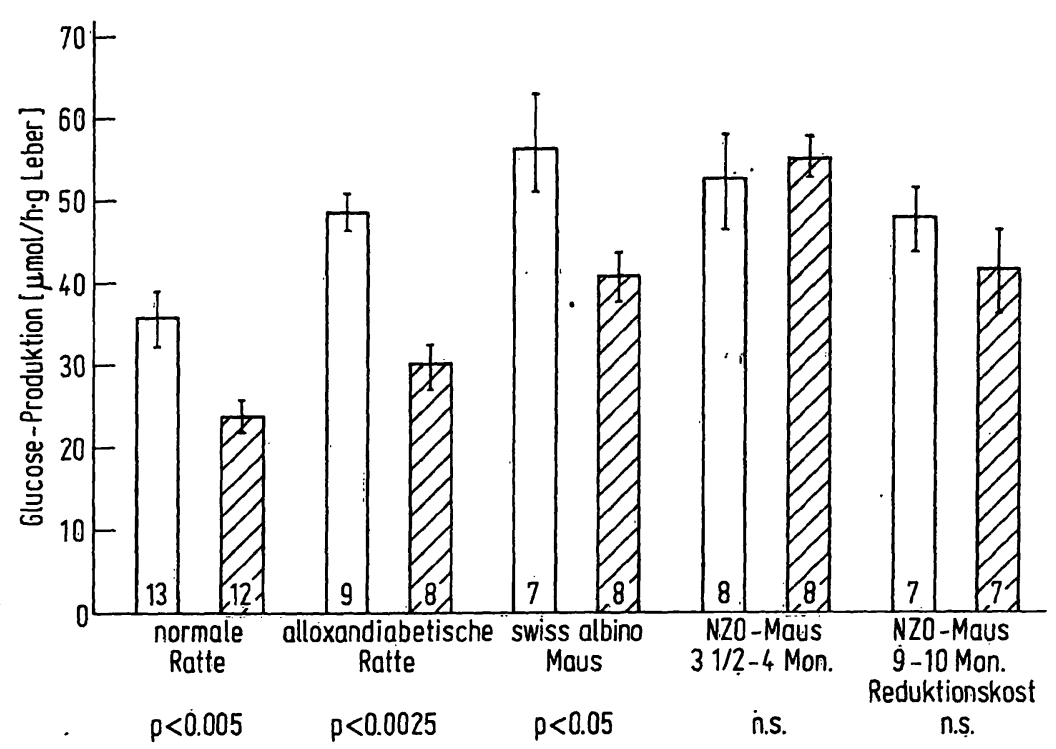

suchten wir den Insulineinfluß an der isoliert perfundierten Leber, am inkubierten epididymalen Fettgewebe, Zwerchfell und Herzmuskel der NZO-Maus vor und nach Gewichtsteduktion.

Wie RUDORFF, et al. $(8,9,10)$ zeigen konnten, hemmt Insulin in der isoliert perfundierten Leber von intakter und alloxandiabetischer Ratte sowie swiss-albino-Maus signifikant die Glucoseproduktion aus $10 \mathrm{mmol} / \mathrm{l}$ LAlanin und normalisiert die signifikant gesteigerte Gluconeogenese der alloxan-diabetischen Ratte (Abb. 3). Dagegen findet sich kein Insulineffekt auf die Glucoseproduktion bei der NZO-Maus einmal im Alter von $31 / 2-4$ Monaten, zum anderen von 9-10 Monaten nach Gewichtsreduktion (Abb. 3). Im Gegensatz zu Ratten und weißer Maus hemmt Insulin ebenfalls nicht die ${ }^{14} \mathrm{CO}_{2}$-Bildung aus [U-14 C]L-Alanin bei den beiden NZO-Maus-Gruppen (Abb. 4). Des weiteren hat Insulin bei Ratte und weißer Maus auf den ${ }^{14} \mathrm{C}$-Einbau in die Perfusatglucose aus [U-14 C]L-Alanin einen msignifikant hemmenden Einfluß, jedoch einen signifikant stimulierenden Effekt auf den ${ }^{14} \mathrm{C}$-Einbau in Leber- und Plasmaproteine $(8,9,10)$. Vergleichsweise läßt auch hier die Leber der NZO-Maus bei dem ${ }^{14} \mathrm{C}$ Einbau in die Perfusatglucose und in das Leberprotein keine Insulinwirkung erkennen (Tab. 2). Bei allen Tierspezies ist ein Insulineffekt auf den ${ }^{14} \mathrm{C}$-Einbau aus [U-14 C]L-Alanin in die Gesamtlipide, auf Harnstoffproduktion und Ketogenese nicht nachzuweisen.

Diese Ergebnisse zeigen einmal, daß Insulin am Modell der isoliert perfundierten Leber von normaler und alloxan-diabetischer Ratte, wie von swiss-albino-Maus einen direkten Einfluß auf den Ĺeberstoffwechsel nimmt, während die Leber der NZO-Maus auch nach Gewichtsreduktion insulinresistent zu sein scheint. Zum anderen ist ersichtlich, daß die Leber der NZOMaus im Vergleich zur swiss-albino-Maus als Kontrollaufweist. tier keine gesteigerte Gluconeogenese aus L-Alanin

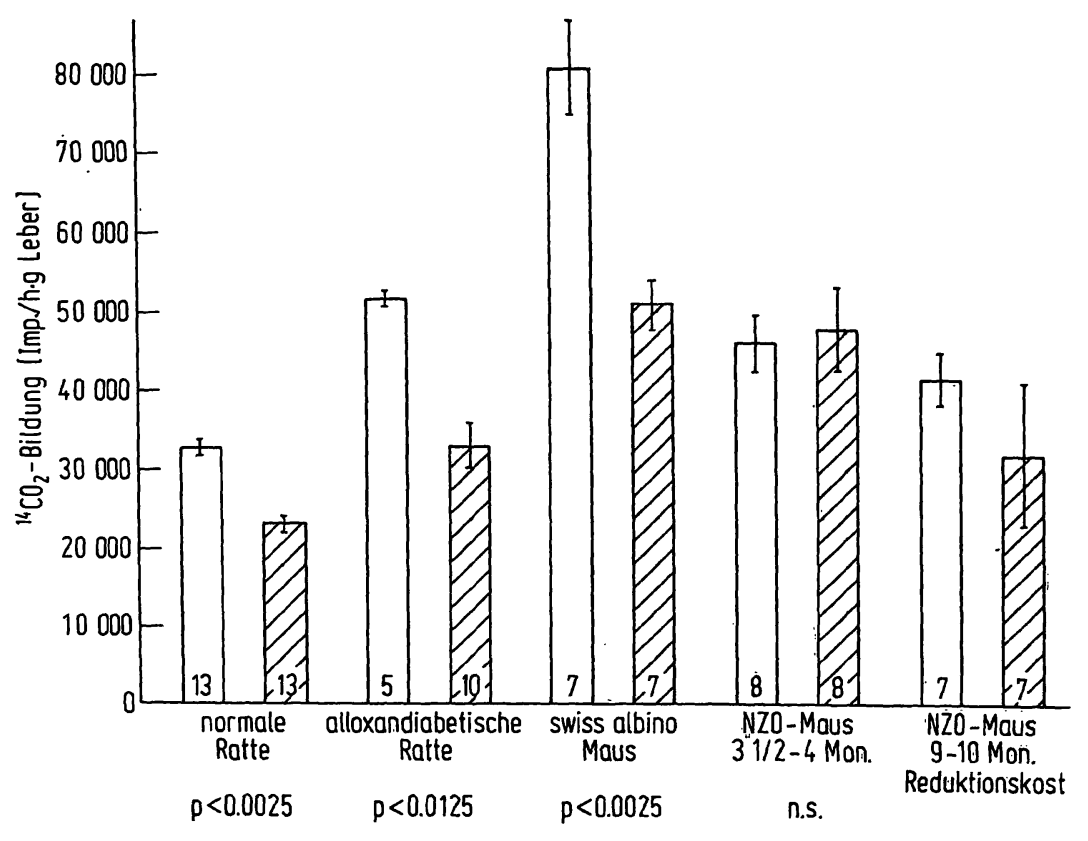

Abb. 4

Einfluß von Insulin auf die ${ }^{14} \mathrm{CO}_{2}$-Bildung aus [U-MCC]LAlanin in der isoliert perfundierten Leber von normaler und alloxandiabetischer Ratte, swiss albino Maus, NZO-Maus. ( $3^{1 / 3}-4$ Monate) und NZO-Maus (9-10 n. s. = nicht signifikant $\square$ Kontrolle" 
Tab. 2

Einfluß von Insulin auf den ${ }^{10} \mathrm{C}$-Einbau aus $10 \mathrm{mmol} / 1$ [U-14C]L-Alanin in die Perfusatglucose (a) und in das Leberprotein (b) in der isoliert perfundierten Leber der NZO-Maus $(\overline{\mathbf{x}} \pm s \overline{\mathbf{x}})$

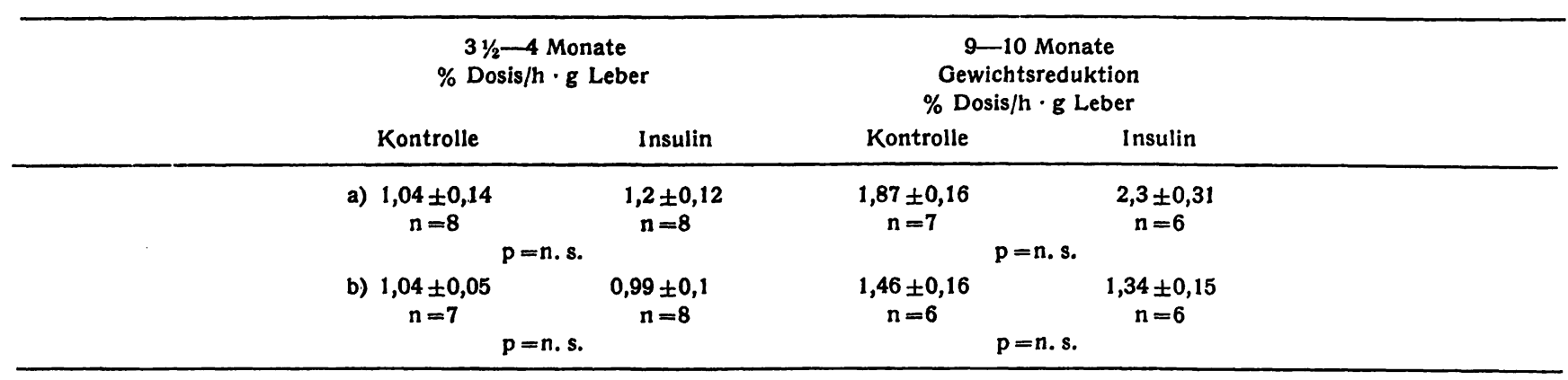

Einfluß von Insulin auf den Glucosestoffwechsel in Muskulatur und Fettgewebe

Der quantitativ wesentliche Glucoseumsatz in Abbängigkeit von Insulin findet in Muskulatur und Fettgewebe statt. Beide Gewebe sollen bei der NZO-Maus nach den in vivo-Untersuchungen von STAUFFACHER und RENOLD (25) relativ unempfindlicher gegen Insulin sein als bei Kontrolltieren, wobei das Zwerchfell resistenter sein soll als das Fettgewebe. Im Gegensatz dazu stehen in vitro Befunde von SNEYD (26), der eine
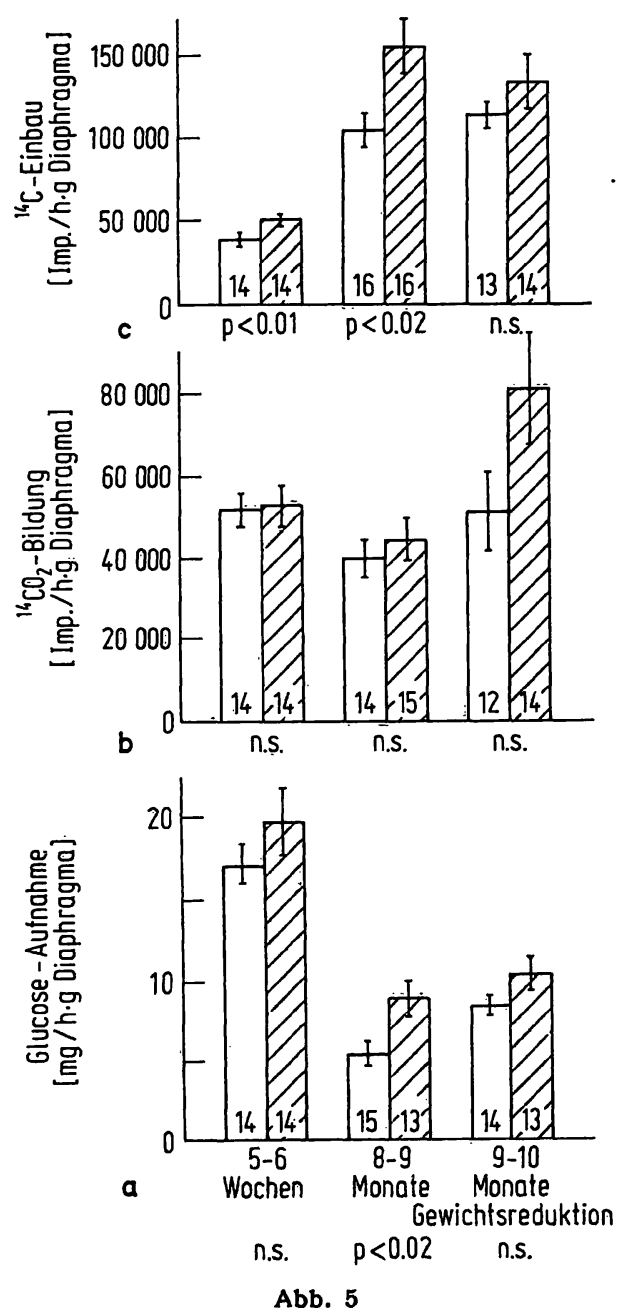

Einfluß von Insulin a) auf die Glucoseaufnahme b) auf die ${ }^{24 \mathrm{CO}_{2}-}$ Bildung aus [U-14C]Glucose $c$ ) auf den ${ }^{4} \mathrm{C}$-Einbau aus [U-1 C] $\mathrm{Clucose}$ in das Glykogen durch das Diaphragma (Bezug: Feuch tgewicht) von NZO-Mäusen im Alter von 5-6 Wochen, 8-9 Monaten und 9-10 Monaten nach Gewichtsreduktion n. s. = nicht signifikant $\square$ Kontrolle M/ Insulin $^{\text {Monaten nach Gewichtsreduktion }}$
Insulinunterempfindlichkeit dieser Gewebe nicht nachweisen konnte.

\section{Muskulatur}

Wir überprüften diese widersprüchlichen Befunde und konnten in vitro am inkubierten Diaphragma von NZO-Mäusen im Alter von 5-6 Wochen, 8-9 Monaten und 9-10 Monaten nach Gewichtsreduktion, gemessen an der Glucoseaufnahme, an $\operatorname{der}{ }^{14} \mathrm{CO}_{2}$ Bildung aus [U-14 $\mathrm{C}$ ]Glucose und dem ${ }^{14} \mathrm{C}$-Einbau aus [U-14C]Glucose in das Glykogen nur in drei Versuchsgruppen einen Insulineffekt nachweisen. Die Glucoseaufnahme aus dem Medium nimmt mit zunehmender Fettsucht basal und insulinstimuliert signifikant ab, um nach Gewichtsreduktion nur in den Basalwerten signifikant anzusteigen, ohne jedoch die Anfangswerte zu erreichen. Die Gewichtsreduktion führt am Zwerchfell nicht zu einer Steigerung der Insulinsensitivität (Abb. 5).

Ein ähnliches Bild ergibt sich bei der Überprüfung der gleichen Parameter an Schnitten von Herzmuskel. Nur in einer Versuchsgruppe ist ein Insulineffekt sichtbar. Auch hier finden wir basal und insulinstimuliert die verminderte Glucoseaufnahme bei den alten, fettsüchtigen Tieren, den basalen Anstieg nach Gewichtsreduktion und ebenfalls keinen stärkeren Insulineffekt nach Gewichtsreduktion (Abb. 6). Hier ist anzumerken, daß nach den Untersuchungen von MorgaN et al. (27) am Herzmuskel alloxandiabetischer Ratten Insulin nur bis zu $85 \%$ den Glucosemembrantransport kontrolliert, so $\mathrm{da}$ B ein insulinunabhängiger Glucoseeinstrom einen normalen Stoffwechsel gewährleisten kann.

\section{Epididymales Fettgenebe}

Am epididymalen Fettgewebe nimmt die Glucoseaufnahme im 8.-9. Monat gleichfalls basal und insulinstimuliert signifikant im Vergleich zu den jungen Tieren $a b$, ein Insulineffekt ist nicht ersichtlich. Im Gegensatz zur Muskulatur erreicht aber nach Gewichtsreduktion die basale Glucoseaufnahme die Anfangswerte und läßt sich darüber hinaus mit Insulin signifikant steigern (Abb. 7). Die ${ }^{14} \mathrm{CO}_{2}$-Produktion ist basal und insulinstimuliert bei jungen und alten Tieren gleich niedrig, nimmt nach Gewichtsreduktion basal signifikant $z u$, um dann unter Insulin eine signifikant 

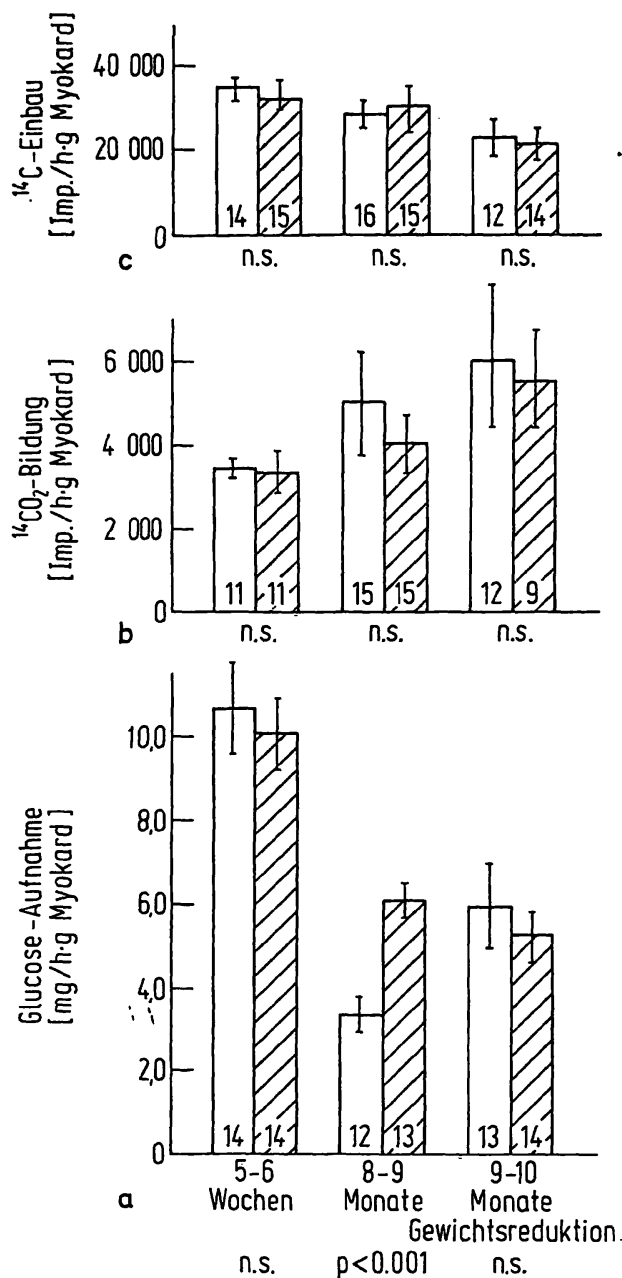

Abb. 6

Einfluß von Insulin a) auf die Glucoseaufnahme, b) auf die ${ }^{14} \mathrm{CO}_{2}$ Bildung aus [U-16 C]Glucose, c) auf den ${ }^{24} \mathrm{C}$-Einbau aus [U-24C]Glucose in das Glykogen durch die Herzmuskulatur (Bezug: Feuchtgewicht) von NZO-Mäusen im Alter von 5-6 Wochen, 8-9 Monaten und 9-10 Monaten nach Gewichtsreduktion

n. s. = nicht signifikant $\square$ Kontrolle $\mathbb{W} /$ Insulin $^{2}$

erhöhte Produktion zu zeigen (Abb. 7). Auch der ${ }^{14} \mathrm{C}$-Einbau in die Gesamtlipide wird mit zunehmendem Alter basal und insulinstimuliert geringer, um dann nach Gewichtsreduktion signifikant anzusteigen. Be- merkenswert ist der Nachweis, daß hier in jeder Gruppe ein signifikanter Insulineffekt besteht, der sich durch Gewichtsreduktion noch weiter stimulieren läßt (Abb. 8). Wir können damit die in vivo durch Staufracher und RENOLD (25) gefundene weitgehende Insulinresistenz von Muskulatur und Fettgewebe in vitro bestätigen und diese Befunde ergänzen, indem basale und insuliristimulierte Glucoseaufnahme mit zunehmender Fettsucht in beiden Geweben abnimmt und indem nach Gewichtsreduktion nur am Fettgewebe eine erhöhte

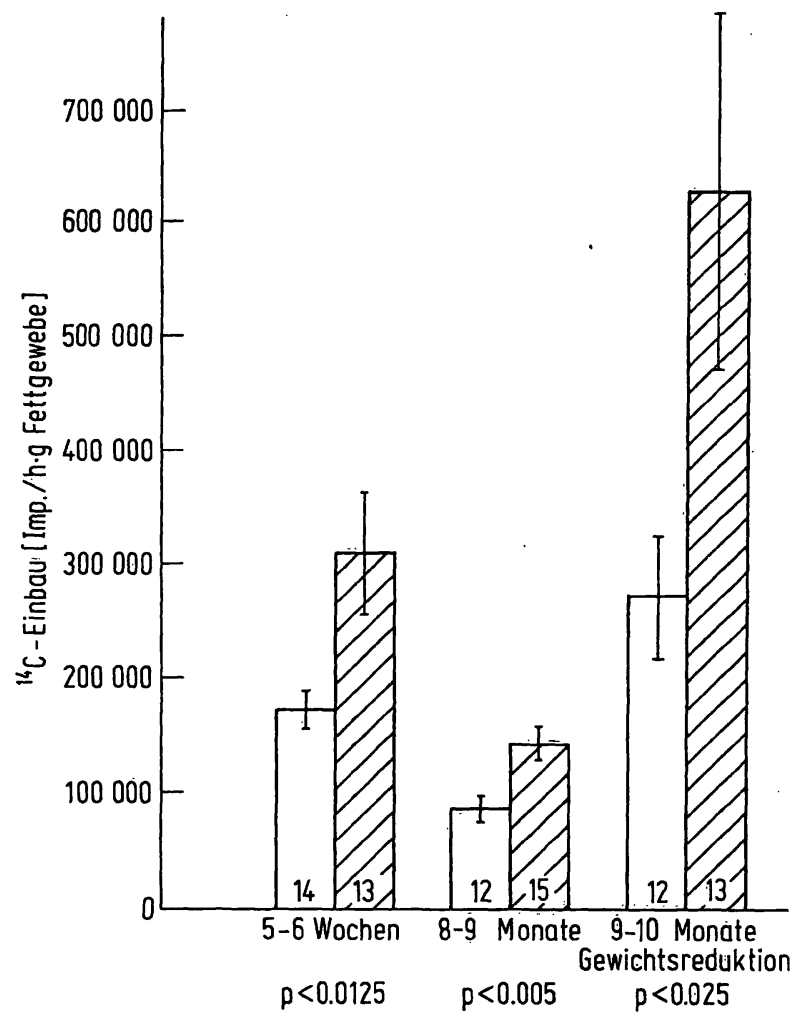

Abb. 8

Einfluß von Insulin auf den "C-Einbau aus [U-14C]Glucose in die Gesamt-Lipide durch epididymales Fettgewebe (Beżug: Feuchtgewicht) von N.ZO-Mäusen im Alter von 5-6 Wochen, 8-9 Monaten und 9-10 Monaten nach Gewichtsreduktion
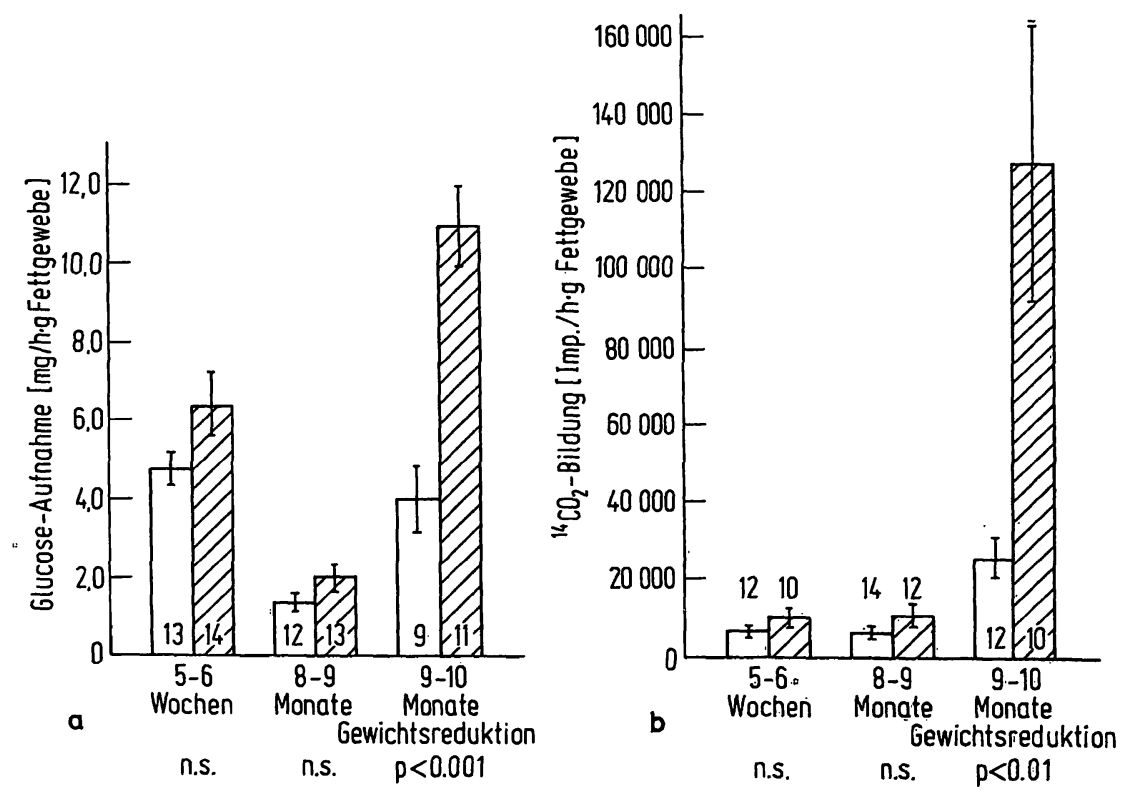

Abb, 7

EinfluB von Insulin a) auf die Glucoseaufnahme b) auf die ${ }^{16} \mathrm{CO}_{2}$-Bildung aus [U-14C]Glucose durch epididymales Fettgewebe (Bezug: Feuchtgewicht) von NZO-Mausen im Alter von 5-6 Wochen, 8-9 Monaten und 9-10 Monaten nach Gewichtsn. s. = nicht signifikant $\square$ Kontrolle $V_{/ E}$ Insulin 
Insulinsensitivität nachzuweisen ist. Das Fettgewebe bietet eine weitere Besonderheit, indem Insulin hier regelmäßig die Lipidsynthese aus Glucose zu stimulieren vermag, so daß wohl der wesentliche Anteil der aufgenommenen Glucose bei nur geringem Abbau zu $\mathrm{CO}_{2}$ auf die Lipogenese entfällt. Vermutlich werden weitere wesentliche Glucosemengen für die Bildung von Lactat und Glykogen bereitgestellt.

Diese Beobachtungen an der NZO-Maus korrelieren gut mit Befunden bei menschlicher Adipositas. So fanden RabINowirz und ZIERLER (21) bei Adipösen mit der Vorderarmmethode eine Insulinunterempfindlichkeit von Muskulatur und Fettgewebe. Ebenso ist in vitro nach Salans et al. (28), Gries (29) und anderen Arbeitsgruppen die Insulinsensitivität des Fettgewebes bei Fettsucht vermindert, was allerdings im Gegensatz zu Befunden von BjörNTORp (30) steht. Nach Gewichtsreduktion fanden SaLANS et al. (28) eine erhöhte Insulinempfindlichkeit des menschlichen Fettgewebes.

\section{Pathogenese des fettsüchtig-hyperglykä- mischen Syndroms}

Es läßt sich somit feststellen, daß der Hyperinsulinämie der NZO-Maus eine zellgebundene Insulinresistenz von Leber, Muskulatur und Fettgewebe gegenübersteht. Für die Störung der Glucosetoleranz (Abb. 1) sind Muskulatur und Fettgewebe als quantitativ wesentliche Orte des insulinabhängigen Glucosemetabolismus verantwortlich. Beim Muskel kommt, wie beschrieben, die Hemmung des Glucoseverbrauches durch die Hyperlipacidämie hinzu. Die führende Rolle scheint aber das Fettgewebe zu spielen. Denn Besserung der Glucosetoleranz sowie erhöhte Insulinsensitivität des Fettgewebes nach Gewichtsreduktion bestätigen die Vorstellungen von SaLANS et al. (28), nach denen Glucosetoleranz in vivo und Insulinsensitivität des Fettgewebes in vitro korreliert sind. Ob die Fettleber der NZO-Maus für die gestörte Glucosetoleranz ebenfalls eine Rolle spielt, kann aus unseren Experimenten nicht beantwortet werden. Nach Madison (31) soll die gesunde Leber maximal 87\% der Glucose bei Glucosebelastung aufnehmen können.

Unsere Untersuchungen zeigen weiterhin, daß die aufgezeigten Störungen durch Gewichtsreduktion partiell reversibel sind und daher nur $z$. T. genetisch determiniert, z. T. aber erworben zu sein scheinen, so daß bei der NZO-Maus für die Stoffwechselanomalie ein rezessiver Gendefekt und Umwelteinflüsse verantwortlich sind.

Zusammenfassend sind für die Pathogenese des hyperglykämischen Syndroms der NZO-Maus neben humoral-metabolischen Faktoren zell- und gewebsgebundene Faktoren von Bedeutung, die die Insulinwirkung verringern. Die Hauptursache dürfte dabei die Insulinresistenz von Fettgewebe, Muskulatur und Leber sein, während eine gesteigerte Gluconeogenese - gemessen an Leberenzymaktivitäten - eine untergeordnete Rolle spielt und vielleicht nur unter dem Einfluß belastender
Faktoren, wie spezieller Diäten, auftritt $(6,12)$. Die isoliert perfundierte Leber jedenfalls läßt eine gesteigerte Gluconeogenese aus L-Alanin vermissen. Und auch der Versuch in vitro an Leberschnitten von NZOMäusen verschiedenen Lebensalters eine gesteigerte Gluconeogenese nachzuweisen, mißlang. Nach $24 \mathrm{~h}$ Futterentzug bildet die Leber von NZO-Mäusen endogen und nach Zusatz verschiedener Substrate, korrigiert auf den initialen Glucose- und Glykogengehalt der Leber - bezogen auf Feuchtgewicht -, die gleiche Menge Glucose plus Glykogen/g.h wie die Leber weißer Mäuse. Die Glucoseproduktion in den Leberschnitten ist dabei signifikant niedriger als in der perfundierten Leber (32). Möglicherweise machen sich bei der sehr geringen Gluconeogeneserate in Leberschnittpräparationen von NZO- und swiss-albino-Maus geschlechts-spezifische Einflüsse geltend. Die Leber der hier verwendeten männlichen Tiere soll eine niedrigere Glucoseproduktion aufweisen als die der weiblichen Tiere (33).

In der Pathogenese der metabolischen Fettsucht der NZO-Maus scheint eine durch Insulin stimulierte verstärkte Lipogenese mit bevorzugter Verwendung von Kohlenhydraten die größte Bedeutung zu haben (vgl. Abb. 8). Die ${ }^{14} \mathrm{CO}_{2}$-Bildung aus ${ }^{14} \mathrm{C}$-Glucose ist niedrig (vgl. Abb: 7) und signifikant geringer als bei Kontrolltieren (34). Eine verzögerte Oxydationsrate von $\left[1-{ }^{14} \mathrm{C}\right]$ Acetat $\mathrm{zu}{ }^{14} \mathrm{CO}_{2}$, die von GugGenHeIM und MAyer (35) als ein Faktor für die Entwicklung des obese syndrome der Bar Harbor obese Maus angesehen wurde, konnte für die NZO-Maus nicht bestätigt werden (34). Wir selbst fanden an der NZO-Maus mit zunehmender Fettsucht in vivo nach intraperitonealer Injektion von [U-14 $\mathrm{C}$ ]Stearinsäure $(1,5 \mu \mathrm{Ci} / 100 \mathrm{~g} \mathrm{Kör-}$ pergewicht) ebenfalls keine signifikanten Unterschiede in der ${ }^{14} \mathrm{CO}_{2}$-Produktion. So atmeten zwei Monate alte Tiere in $2 \mathrm{~h} 22,07 \pm 2,26 \% \quad(\mathrm{n}=9)$ der injizierten Dosis ab im Vergleich zu 6 Monate alten Tieren mit $18,52 \pm 1,66 \%(n=9)$. Entsprechend zeigte der Einbau der $\left[\mathrm{U}^{14} \mathrm{C}\right]$ Stearinsäure in die Gesamtlipide der Leber keine Unterschiede. Die jungen Tiere bauten in $2 \mathrm{~h} 6,27 \pm 2,75 \%(\mathrm{n}=9)$, die älteren Tiere 7,24 $\pm 1,49 \%(n=9)$ der injizierten Dosis in $100 \mathrm{mg}$ Gesamtlipide der Leber ein. Bestätigt werden diese Befunde in vitro an der isoliert perfundierten Leber $(0,5 \mu \mathrm{Ci}$ [U_-14 C]Stearinsäure/Perfusion). In der Leber der zwei Monate alten Tiere fanden sich nach $1 \mathrm{~h} 15,25$ $\pm 1,91 \%(n=9)$, in der Leber der 6 Monate alten Tiere $15,29 \pm 2,19 \%(n=9)$ der Dosis in $100 \mathrm{mg}$ Gesamtlipide wieder.

In den vorliegenden tierexperimentellen Untersuchungen konnten zahlreiche Parallelen zum menschlichen Reifediabetes aufgezeigt werden. Úberträgt man das Modell der NZO-Maus auf den Menschen, so entspricht es dem fettsüchtigen Patienten, der eine gestörte Glucosetoleranz bei gleichzeitiger Hyperinsulinämie aufweist, ohne daß es bisher zu einer Erschöpfung der Inselzellfunktion gekommen ist (vgl. 36). 


\section{Literatur}

1. Gries, F. A. \& Oberdisse, K. (1970), Deut. Med. Wochenschr. 95, 727-734. - 2. JosiIn, L. P., Root, H. F., WhIte, P. \& Marbl, A. (1959), The Treatment of Diabetes mellitus; 10th Ed. Philadelphia. - 3. DAwEKE, H., LIEBERMEISTER, H., GRÜNEKLEE, D. \& Oberdisse, K. (1969), Med. Welt 20 N. F., 1872-1876. 4. Renold, A. E. (1967), Verh. Deut. Ges. Inn. Med. 72, 839 bis 850. - 5. BieLsChowsKY, M. \& BieLsChOWSKY, F. (1953), Proc. Univ. Otago med. Sch. 31, 29-31. - 6. HuchzermeYer, H. \& Staib, W. (1972), im Druck. - 7. Rudorff, K. H., HuCHZERMEYER, H. \& STAIB, W. (1970), unveröffentlicht. - 8. RUDORFF, K. H., WindeCK, R., HuchzermeYer, H. \& Starb, W. (1971), 5th Meeting of the European Association for the study of the liver, Berne 1970, Digestion 4, 174. - 9. RudorfF, K. H., Albrecht, G. \& Starb, W. (1970a), Hoppe-Seyler's Z. Physiol. Chem. 351, 975-982. - 10. RUDORFF, K. H., HUCHZERMEYER, H., WINDECK, R. \& Staib, W. (1970b), Europ. J. Biochem. 16, 481-486. 11. Herberg, L., Major, E., Hennings, U., Grüneklee, D., FreYTAG, G. \& GRIES, F. A. (1970), Diabetologia 6, 292-299. 12. Willms, B., Ben-AMr, P. \& Söling, H. D. (1970), Horm. Metab. Res. 2, 135-141. - 13. Hoffman, W. S. (1937). J. Biol. Chem. 120, 51-55. - 14. SterN, G. \& BässLER, K. H. (1968), diese Z. 6, 27-30. - 15. McLean, J. R., Cohn, G. L., BRANDT, J. K. \& Srmpson, M. V. (1958), J. biol. Chem. 233, 657-663. 16. Folch, J., Lees, M. \& Stanley, G. H. S. (1957), J. Biol. Chem. 226, 497-509. - 17. WalaAs, O. \& WalaAs, E. (1950), J. Biol. Chem. 187, 769-776. - 18. Rosenthal, H. L., Pfluke,
M. L. \& Calrerami, J. (1959), Clin. Chim. Acta 4, 329-333. 19. KREUTZ, F. H. (1962), Klin. Wochenschr. 40, 362-363. 20. ITAYA, K., Ur, M. (1965), J. Lipid Res. 6, 16-19. - 21. Rabinowitz, D. \& ZierLeR, K. L. (1962), J. Clin. Invest. 41, 2173-2181. - 22. BielschowsKx, M. \& BrelsChowsKY, F., Aust. J. (1956), J. Exp. Biol. Med. Sci. 34, 181-198. - 23. LARKINS, R. G. (1971), Diabetologia 7, 302-307. - 24. RANDLE, P. J., Gariand, P. B., Hales, C. N. \& Newsholme, E. A. (1963), Lancet I, 785-789. - 25. Stauffacher, W. \& Renold, A. E. (1969), Amer. J. Physiol. 216, 98-105. - 26. SNEYr, I. G. T. (1964), J. Endocrinol. 28, 163-172. - 27. MoRGaN, H. E., Cadenas, E., Regen, D. M. \& Park, C. R. (1961), J. Biol. Chem. 236, 262-268. - 28. Salans, C. B., KnImtLe, J. L. \& Hirsch, J. (1968), J. Clin. Invest. 47, 153-165. - 29. Gries, F. A. (1970), Adipose Tissue. Regulation and Metạbolic Functions, 167-171, Stuttgart. - 30. BJöRNTORP, P. (1970), Diabetologia 6, 654. - 31. Madison, L. L. (1965), Internat. Diabetes Federation V. Congr. Exc. Med. Foundation. - 32. HuchzermeXer, H. \& STAIB, W. (1970), unveröffentlicht. - 33. KREBS, H. A., Norton, B. M. \& HEMs, R. (1966), Biochem. J. 101; 607-617. - 34. Subrahmanyam, K. (1960), Biochem. J. 76, 548-556. - 35. Guggenfeim, J. \& Mayer, J. (1952), J. Biol. Chem. 198, 259 bis 265. - 36. Stauffacher, W. \& ORCI, L. (1970), 76. Tagung der Deutschen Gesellschaft für Innere Medizin, 6.-9. April 1970, Wiesbaden.
Prof. Dr. W. Staib

Institut für Physiologische Chemie II der Universität Düsseldorf 4000 Düsseldorf

Moorenstr. 5 